\title{
Economics
}

The Open-Access, Open-Assessment E-Journal

Vol. 12, 2018-64 | October 23, 2018 | http://dx.doi.org/10.5018/economics-ejournal.ja.2018-64

\section{Dynamic pricing for inventories with reference price effects}

\author{
Régis Chenavaz and Corina Paraschiv
}

\begin{abstract}
This article presents a dynamic pricing model of a retailer selling an inventory, accounting for consumer behavior. The authors propose an optimal control model, maximizing the intertemporal profit with consumers sensitive to the selling price and to a reference price. The optimal dynamic pricing policy is solved with Pontryagin's maximum principle with a structural (general) demand function. The authors obtain an original pricing rule, which explicitly accounts for the impact of price and inventory on future profits. The dynamics of price do not have to imitate the dynamics of the reference price. Instead, the dynamics of price are tied to opposing effects linked to this reference price. The authors also discuss managerial implications with regards to behavioral pricing policies.
\end{abstract}

JEL C61 D03 D40 M21 M37

Keywords Dynamic pricing; inventory; reference price; behavioral pricing; optimal control

\section{Authors}

Régis Chenavaz, Kedge Business School, Aix-Marseille School of Economics, CNRS \& EHESS, France, regis.chenavaz@kedgebs.com

Corina Paraschiv, Paris Descartes University \& Institut Universitaire de France, Paris, France

Citation Régis Chenavaz and Corina Paraschiv (2018). Dynamic pricing for inventories with reference price effects. Economics: The Open-Access, Open-Assessment E-Journal, 12 (2018-64): 1-16. http://dx.doi.org/10.5018/economics- ejournal.ja.2018-64 


\section{Introduction}

Dynamic pricing is a common practice where sellers adjust prices for products or services based on current demand. Developed initially by the airline industry with yield management, dynamic pricing is now a wide-spread phenomenon (Den Boer, 2015). Recent technological evolutions concerning real-time access to demand-related data (big data), real-time data processing, pricing algorithm automatization (allowing for real-time computation of optimal prices), and new technologies to implement frequent price changes (smart labels) contribute to dynamic pricing diffusion in the retailing industry. A retailer is traditionally confronted with inventory management issues, for which price is a main control (Goyal and Giri, 2001; Elmaghraby and Keskinocak, 2003; Bakker et al., 2012). New dynamic pricing models need to be developed that also address inventory management issues together with actual consumer behavior. This point motivates our research.

In this article, we study the dynamic pricing policy of a retailer selling an inventory when the reference price of consumers plays a role. In the modeling, we assume a fixed inventory without possibility of replenishment. This assumption describes well a wide range of retail products: markdown products, products at the end of their life cycle with excess inventory, or products with a short life cycle and long procurement delays (Goyal and Giri, 2001; Elmaghraby and Keskinocak, 2003; Bakker et al., 2012). In such situations, the retailer needs to make pricing decisions given a fixed amount of inventory and a limited time horizon. Concerning consumer behavior, we posit a reference price effect. Consumer decisions to purchase a product depend not only on the retail (or selling) price, but also on its reference price. The reference price is a psychological price used as a benchmark against which consumers compare the retail price (Sorger, 1988; Mazumdar et al., 2005). A retail price below the reference price is perceived by consumers as a gain, which increases demand. Conversely, a retail price above the reference price is perceived as a loss, reducing demand (Kopalle et al., 1996; Popescu and Wu, 2007; Nasiry and Popescu, 2011).

The literature on behavioral dynamic pricing and inventory management informs this research. The dynamic pricing research stream that formally accounts for reference dependence was initiated decades ago by Sorger (1988) and Kopalle and Winer (1996). Building on this approach, Kopalle et al. (1996) and Fibich et al. (2003) consider asymmetric reference effects. The joint study of reference effects with inventories is more recent, with Taudes and Rudloff (2012) proposing a two-period model. Stochastic demand and stochastic inventory are introduced in Cao et al. (2012) and Li et al. (2015), respectively. Dye and Yang (2016) and Xue et al. (2016) study the optimal pricing of deteriorating items in the presence of a reference price. Demand depending on inventory is analyzed by Lu et al. (2016) and Hsieh and Dye (2017). Previous research use parametric demand functions. Güler et al. (2017) look at both dynamic pricing and inventory issues, offering numerical simulations. We adopt the approach of Popescu and Wu (2007), Chenavaz (2016), and Chenavaz (2017), using structural (general) demand functions, which enables us to derive more general results.

Building on the aforementioned studies, we propose a dynamic pricing policy of a nonperishable inventory with a reference-dependent demand. To the best of our knowledge, this is the first attempt to integrate a general demand function-assuming little restriction on consumer behavior-in inventory management. Analytical results show how the price setting is tied to 
inventory and reference price considerations. They also characterize how the dynamics of the selling price are tied to the dynamics of the reference price. A firm ignoring the psychological element would set an inadequate pricing policy, thus losing profit. The integration of consumer behavior provides a greater understanding of firm dynamic pricing strategies.

\section{Modeling Assumptions}

We study the optimal intertemporal pricing strategy of a retailer in a monopoly situation. The retailer sells an inventory during the fixed and finite planning period $T>0$. The time $t \in[0, T]$ is continuous.

\subsection{Consumer Reference Price}

To decide on a purchase decision at time $t$, a consumer compares the current retail price $p(t) \geqslant 0$ with a reference price $r(t) \geqslant 0$. The reference price represents a benchmark informing the customers about the opportunity of a transaction (Sorger, 1988; Mazumdar et al., 2005). If $p(t)>r(t)$, the consumer perceives a loss; he is less likely to buy. Conversely, if $p(t) \leqslant r(t)$, the consumer perceives a gain; he is more likely to buy.

The reference price is usually formalized through a weighted average of past product prices (Sorger, 1988; Kopalle et al., 1996; Kopalle and Winer, 1996; Fibich et al., 2003). We define the reference price at time $t, r(t)$ with an exponentially decaying function: $r(t)=e^{-\beta t}\left(r_{0}+\right.$ $\left.\beta \int_{0}^{t} e^{\beta s} p(s) d s\right)$ where $r_{0}$ is the initial reference price at time $t=0$, and $\beta \geqslant 0$ is the continuous forgetting (or adjustment speed) parameter. Differentiate $r(t)$ with respect to time $t$ :

$$
\frac{d r(t)}{d t}=\beta(p(t)-r(t)), \text { with } r(0)=r_{0} .
$$

Equation (1) states that the variation of the reference price increases with the adjustment speed $\beta$ and with the difference between the retail price $p(t)$ and the reference price $r(t)$.

\subsection{General Reference-Dependent Demand}

The demand $D \geqslant 0$ depends on the retail price $p$ and the reference price $r$. We consider in this article a general reference-dependent demand function $D=D(p(t), r(t))$. This general form offers the advantage of allowing for nonlinearities when capturing dynamics related to reference price variations (Popescu and $\mathrm{Wu}, 2007)$. An even more general demand function could include time dependency with $D=D(p(t), r(t), t)$, in the vein of Schlosser (2015), Schlosser (2016), and Güler et al. (2017).

For brevity and in order to simplify equations, function parameters will be further omitted, if there is no confusion. The demand function is twice continuously differentiable, satisfying the following conditions

$$
\frac{\partial D}{\partial p}<0, \frac{\partial D}{\partial r}>0, \frac{\partial^{2} D}{\partial p \partial r} \leqslant 0
$$


The first condition captures a price effect $(\partial D / \partial p<0)$ by imposing that the demand decreases with the retail price. The second condition refers to a direct reference effect $(\partial D / \partial r>0)$, where the demand increases with the reference price. The third condition captures an indirect reference effect $\left(\partial^{2} D / \partial p \partial r \leqslant 0\right)$. That is, the decrease of the demand due to an increase in price is higher when the reference price is high.

In line with the general demand approach initiated by Popescu and $\mathrm{Wu}$ (2007) and followed by Chenavaz (2016), condition (2) imposes relatively weak restrictions on the impact of retail price and reference price on demand. Also such assumptions cope with the two main cases of demand separability.

- Additively separable case $D=f(p)+g(r)$, of which the classical linear demand function $D=\alpha-\delta p-\gamma(p-r)$ is an example. Such a function is widely used, for instance by Sorger (1988), Kopalle et al. (1996), Kopalle and Winer (1996), Dye and Yang (2016), Xue et al. (2016), and Hsieh and Dye (2017). The implication of additive separability is to impede any indirect reference effect as $\partial^{2} D / \partial p \partial r=0$. Such an implication simplifies the tractability of any model and thus its analysis, though at a cost of lesser generality of the results.

- Multiplicatively separable case $D=f(p) g(r)$, for which the Cobb-Douglas (isoelastic) demand function $D=\alpha p^{-\delta} r^{\gamma}$ represents an instance. Because of opposite monotonies, the indirect reference effect writes $\partial^{2} D / \partial p \partial r<0$, enriching the analysis with a cross effect at the demand level. Popescu and $\mathrm{Wu}$ (2007) provide greater details on the properties of the demand functions.

\subsection{Inventory}

Firm inventory at $t$ writes $I(t) \geqslant 0$. We exclude the possibility of replenishment as in Taudes and Rudloff (2012), Dye and Yang (2016), Xue et al. (2016), and Hsieh and Dye (2017). The lifetime $T$ of the inventory is fixed, that is, there is a predeterministic selling period (say several months or seasons). Inventory decrease depends on previous sales and items do not perish over time. This situation corresponds to class (1) in the great survey by Bakker et al. (2012, p. 276). Formally, the inventory at time $t$, denoted $I(t)$, is given by

$$
I(t)=I_{0}-\int_{0}^{t} D(p(s), r(s)) d s, \text { with } I(0)=I_{0} \text { and } I(T)=0 .
$$

The inventory at time $t$ equals the initial inventory minus the cumulative demand during the period 0 to $t$. The differentiation of $I(t)$ with respect to time $t$ yields

$$
\frac{d I(t)}{d t}=-D(p, r)
$$

Equation (4) states that inventory variation at time $t$ decreases with current demand. Also inventory does not decrease autonomously as in the case of perishable items. 


\subsection{Current Profit}

The current profit of the retailer $\pi \in \mathbb{R}$ corresponds to the difference between revenues and costs. Revenues depend on price and demand. Costs depend on inventory level $I$ and unitary inventory cost $h$. Thus, the profit of the retailer writes

$$
\pi(p(t), r(t))=p(t) D(p(t), r(t))-h I(t) .
$$

Substituting (3) in (5) gives

$$
\pi(p(t), r(t))=p(t) D(p(t), r(t))-h\left(I_{0}-\int_{0}^{t} D(p(s), r(s)) d s\right) .
$$

Equation (6) states that current profit of the retailer increases with price, current demand, and also with past demand, which, by reducing current inventory, reduces current inventory costs.

\section{Dynamic Pricing Policy}

The retailer, in a monopoly situation, is modeled within an optimal control framework. Table 1 provides the notations.

Table 1: Notations

\begin{tabular}{ll}
\hline$T$ & $=$ fixed terminal time of the planning horizon, \\
$\rho$ & $=$ interest rate, \\
$p(t)$ & $=$ selling price at time $t$ (control variable), \\
$r(t)$ & $=$ reference price at time $t$ (state variable), \\
$I(t)$ & $=$ inventory at time $t$ (state variable), \\
$D(p, r)$ & $=$ current demand, \\
$\frac{d r(t)}{d t}$ & $=\beta(p-r)=$ reference point dynamics at time $t$, \\
$\frac{I(t)}{d t}$ & $=-D(p, r)=$ inventory dynamics at time $t$, \\
$\lambda_{r}(t)$ & $=$ current-value adjoint variable for $r$ at time $t$, \\
$\lambda_{I}(t)$ & $=$ current-value adjoint variable for $I$ at time $t$, \\
$h$ & $=$ inventory cost, \\
$\pi(p, r)$ & $=p D-h I=$ current profit, \\
$H(p, a, r, \lambda)$ & $=$ current-value Hamiltonian. \\
&
\end{tabular}

\subsection{General Case}

The retailer maximizes the intertemporal profit by setting the optimal pricing, while accounting for reference price and inventory dynamics. With the discount rate $\rho \geqslant 0$, the problem of the retailer 
writes

$$
\begin{aligned}
& \max _{p(t) \geqslant 0} \int_{0}^{T} e^{-\rho t} \pi(t) d t, \\
& \text { subject to } \\
& \frac{d r(t)}{d t}=\beta(p(t)-r(t)), \text { with } r(0)=r_{0}, \\
& \frac{d I(t)}{d t}=-D(p, r), \text { with } I(0)=I_{0} .
\end{aligned}
$$

The maximum principle allows solving the dynamic optimization problem. With the shadow price of the reference price $\lambda_{r}(t)$ and the shadow price of the inventory $\lambda_{I}(t)$, the current-value Hamiltonian $H$ is

$$
H\left(p, r, \lambda_{r}, \lambda_{I}\right)=p D(p, r)-h\left(I_{0}-\int_{0}^{t} D(p, r) d s\right)+\lambda_{r} \beta(p-r)-\lambda_{I} D(p, r) .
$$

The interpretation of current-value Hamiltonian $H$ is as follows: $H$ sums (1) current profit $p D(p, r)-h I$ and (2) future profits due to reference point variation $\lambda_{r} \beta(p-r)$ and inventory variation $-\lambda_{I} D$. $H$ thus represents the instantaneous total (intertemporal) profit at time $t$.

The maximum principle imposes the dynamics of $\lambda_{I}$ to satisfy

$$
\frac{d \lambda_{I}}{d t}=\rho \lambda_{I}-\frac{\partial H}{\partial I}=\rho \lambda_{I}+h, \text { with } \lambda_{I}(T)=0 .
$$

Integrating (8) with respect to time gives ${ }^{1}$

$$
\lambda_{I}(t)=-\int_{t}^{T} e^{-\rho(s-t)} h d s .
$$

By computing the integral, (9) becomes

$$
\lambda_{I}(t)=-\left(1-e^{-\rho(T-t)}\right) \frac{h}{\rho} .
$$

From (9) we derive $\lambda_{I}(t) \leqslant 0$, meaning that the shadow price of the inventory is negative over the planning period. Thus, an inventory is always costly for the retailer. Note that $\lambda_{I}(t)$ is not reference dependent. Instead, it depends on the unitary inventory cost $h$, the interest rate $\rho$, and the remaining selling period $T-t$. Moreover, the function increases over time. The rational is the following: At the beginning of the selling period, the inventory is large, and the high inventory costs reduce the profit of the retailer. But, as time passes, the inventory is lower, and the smaller inventory costs exert less influence on profit.

The maximum principle dictates the dynamics of $\lambda_{r}$

$$
\frac{d \lambda_{r}}{d t}=\rho \lambda_{r}-\frac{\partial H}{\partial r}=(\rho+\beta) \lambda_{r}-\left(p+h-\lambda_{I}\right) \frac{\partial D}{\partial r}, \text { with } \lambda_{r}(T)=0 .
$$

1 The proof of (9) is in Appendix A.1 
Integrating (11) with respect to time gives ${ }^{2}$

$$
\lambda_{r}(t)=\int_{t}^{T} e^{-(\rho+\beta)(s-t)}\left(p+h-\lambda_{I}\right) \frac{\partial D}{\partial r} d s .
$$

Because $\lambda_{I}(t) \leqslant 0$, then $\left(p+h-\lambda_{I}\right)>0$. Thus and recalling $\frac{\partial D}{\partial r} \geqslant 0$, we conclude $\lambda_{r} \geqslant 0$. At any time $t$, an increase in the reference price augments the intertemporal profit. That is, a higher reference price is associated with larger intertemporal profits of the retailer (consumers are more likely to purchase).

We confine our interest to an interior solution for the price, provided it exists. The necessary and sufficient first- and second-order conditions for $p$ impose for all $t \in(0, T)$ :

$$
\begin{aligned}
& \frac{\partial H}{\partial p}=0 \Longrightarrow(1+h) D+p \frac{\partial D}{\partial p}+\beta \lambda_{r}-\frac{\partial D}{\partial p} \lambda_{I}=0 . \\
& \frac{\partial^{2} H}{\partial p^{2}}<0 \Longrightarrow-(2+h) \frac{\partial D}{\partial p}-\left(p-\lambda_{I}\right) \frac{\partial^{2} D}{\partial p^{2}}>0 .
\end{aligned}
$$

The first-order condition on price (13a) yields a first result. ${ }^{3}$

Proposition 1. At any time, price setting is such that

$$
-\frac{\partial D}{\partial p} \frac{p}{D}=1+h+\frac{1}{D}\left(\beta \lambda_{r}-\frac{\partial D}{\partial p} \lambda_{I}\right)
$$

for all $t$ in $[0, T]$ with $\lambda_{r} \geqslant 0$ and $\lambda_{I} \leqslant 0$ given by (10) and (12).

Proof. Divide (13a) by $D$ and rearrange.

Proposition 1 shows how the pricing strategy of a retailer integrating inventory and reference price considerations differs from the pricing of a standard static monopoly, for which the price elasticity of demand is unitary $(-(\partial D / \partial p) /(p / D)=1)$. When the price elasticity of demand is greater than one, the demand is called elastic, and the retailer loses market power. Alternatively, when the price elasticity of demand is lower than one, the demand is called inelastic.

According to Proposition 1, the price elasticity of retailer demand relies on three competing effects: one tied to current profit and two tied to the dynamics of future profits. The presence of inventory cost $h$ has a direct positive effect on the price elasticity of the demand. The demand becomes elastic, reflecting a loss of market power for the retailer because of the reduction of the current profit due to increased current costs.

A greater reference effect also weakens the market power of the firm. The past prices set by the retailer have more influence on the current retail price. That is, the retailer has less freedom to set prices, and the demand becomes more elastic. Formally, the price elasticity of demand increases when the shadow price of the reference price $\lambda_{r}$ increases. A dynamic effect of the inventory, tied to $\lambda_{I}$, is also observed. Indeed, due to the future costs of the inventory, the retailer has an incentive to sell more in the present. This strategy enables diminishing future inventory, reducing

\footnotetext{
2 The proof of (9) is in Appendix A.2

3 The proof of (13a) is obvious and the proof of (13b) appears in Appendix A.3.
} 
the associated costs, and increasing future profits. Thus, the shadow price of the inventory exerts a negative impact, reducing the price elasticity of the demand. Eventually, the larger the demand $D$, the lower is the impact of the shadow prices of reference price and inventory on price setting. Depending on the relative strength of these competing effects, the pricing policy is such that the demand is inelastic, unitary, or elastic.

The managerial implications are straightforward. A firm considering only the current profit, because it ignores lasting reference price and inventory effects, sets a price such that the demand is elastic $(1+h)$. By contrast, a firm integrating current and future profits, because it accounts for lasting effects, may charge a price for which the demand is inelastic (if the lasting inventory effect outweighs the lasting reference effect and the inventory cost effect).

Proposition 1, informing about the price at any time $t$ offers preliminary insights. As such, it characterizes the optimal relationship between the price level on one side and inventory cost and dynamics effects on the other side. Yet, it says nothing about the conditions under which the optimal price goes up or down after a change in the inventory cost and dynamics elements. That is, Proposition 1 maintains silence about any causal relationship.

Proposition 1 has to hold during the whole selling period. To maximize the intertemporal profit, marginal revenue variations must balance marginal cost variations. The relationship between the dynamics of price and the intertemporal elements is made explicit by the time differentiation of the first-order conditions (13a). This time decomposition method has been used to show explicit causality (see, for example, Kalish (1983) and Chenavaz and Jasimuddin (2017)).

The first-order condition on price (13a) must hold at any time $t \in[0, T]$. Its decomposition with respect to time supports the following result.

Proposition 2. Over time, price dynamics are such that

$$
\begin{aligned}
& \frac{d p}{d t}(\underbrace{-(2+h) \frac{\partial D}{\partial p}-\left(p-\lambda_{I}\right) \frac{\partial^{2} D}{\partial p^{2}}}_{+})=\frac{d r}{d t}(\underbrace{(1+h) \frac{\partial D}{\partial r}}_{+}+\underbrace{\left(p-\lambda_{I}\right) \frac{\partial^{2} D}{\partial p \partial r}}_{-}) \\
& +\beta(\underbrace{(\rho+\beta) \lambda_{r}}_{+} \underbrace{-\left(p+h-\lambda_{I}\right) \frac{\partial D}{\partial r}}_{-})-\frac{\partial D}{\partial p}(\underbrace{\rho \lambda_{I}+h}_{+}),
\end{aligned}
$$

for all tin $(0, T)$ with $\lambda_{r} \geqslant 0$ and $\lambda_{I} \leqslant 0$ given by (10) and (12).

Proof. Differentiate (13a) with respect to time $t$. The detailed proof is in Appendix A.4.

Proposition 2 captures the evolution of the optimal price over time. The second order condition (13b) guarantees that, on the left side of Proposition 2, the second factor is positive. On the rightside of Proposition 2 appear three additively separable terms. The first term relates to reference price dynamics, the second to the adjustment speed of the reference price, and the third to the price sensitivity of demand. For the first two terms the impact is ambiguous as they result from two 
competing effects. The third term has a positive impact, ${ }^{4}$ informing that, counterintuitively, the price increases with the price sensitivity of consumers.

Also, it may not be beneficial for the firm to start with extremely high prices, even though such high price would increase the reference price, stimulating the demand afterwards. Indeed, the firm must pay holding cost for the inventory. Such holding costs mitigate the interest of starting with a high price, as a low initial demand is costly in terms of remaining holding costs.

The lesson is that, for a general demand, the dynamics of price relate to the reference price level and adjustment and also to the price sensitivity of consumers, though, the total effect is ambiguous: No simple penetration or skimming pricing policy appears. To obtain more conclusive results, we consider more specific cases, trading off generality for strength of the results.

\subsection{Special Cases}

\section{Absence of Inventory Cost}

When there is no inventory cost $(h=0)$, the shadow price of inventory equals zero (substituting $h=0$ in (10) yields $\lambda_{I}=0$ ). Substituting $h=0$ and $\lambda_{I}=0$ in Propositions 1 and 2 yields

$$
\begin{aligned}
& -\frac{\partial D}{\partial p} \frac{p}{D}=1+\frac{\beta \lambda_{r}}{D}, \\
& \frac{d p}{d t}(\underbrace{-2 \frac{\partial D}{\partial p}-p \frac{\partial^{2} D}{\partial p^{2}}}_{+})=\frac{d r}{d t}(\underbrace{\frac{\partial D}{\partial r}+p \frac{\partial^{2} D}{\partial p \partial r}}_{+})+\beta(\underbrace{(\rho+\beta) \lambda_{r}}_{-} \underbrace{-p \frac{\partial D}{\partial r}}_{-}) .
\end{aligned}
$$

This case rules out the opposing effects due to inventory. Only remains the reference price effect, which is strictly positive. Consequently, the retailer sets a price such that the price elasticity of demand is elastic $\left(-\frac{\partial D}{\partial p} \frac{p}{D}>1\right)$. That is, because of the lasting effect of past prices, the retailer enjoys less market power in price setting. Also, the selling price may exhibit a decreasing or increasing pattern, or a mix of both. Note that the optimal pricing rules above matches the rules in Chenavaz (2016, Propositions 1 and 2), which also characterize a dynamic pricing policy with a reference-dependent demand. More precisely, our case with a fixed inventory and no inventory cost corresponds to their case, which does not consider production cost and inventory issues.

\section{Absence of Reference Adjustment}

When the reference price does not adjust over time $(\beta=0)$, it is constant over time $(d r / d t=0)$ and equals the reference price at time 0: $r(t)=r_{0}$. Substituting $\beta=0$ in Propositions 1 and 2 yields

4 The proof is straightforward. Substituting $\lambda_{I}$ from (10) yields $\rho \lambda_{I}+h=-h\left(1-e^{-\rho(T-t)}\right)+h=h e^{-\rho(T-t)}$, which is positive. That is, $\rho \lambda_{I}+h>0$. Further, demand conditions (2) impose $\frac{\partial D}{\partial p}<0$, enabling derivation of the positivity of the third factor: $-\frac{\partial D}{\partial p}\left(\rho \lambda_{I}+h\right)>0$. 


$$
\begin{aligned}
& -\frac{\partial D}{\partial p} \frac{p}{D}=1+h+\frac{-\frac{\partial D}{\partial p} \lambda_{I}}{D}, \\
& \frac{d p}{d t}(\underbrace{-(2+h) \frac{\partial D}{\partial p}-\left(p-\lambda_{I}\right) \frac{\partial^{2} D}{\partial p^{2}}}_{+})=\underbrace{-\frac{\partial D}{\partial p}\left(\rho \lambda_{I}+h\right)}_{+} .
\end{aligned}
$$

The first equation informs that when the reference price does not adjust, the price elasticity of demand decreases compared to the general case treated in Proposition 1 (the term $\frac{\beta \lambda_{r}}{D}$ was positive and is now null). This suggests that, when the reference price is constant, the firm benefits from more market power. Indeed, past prices do not affect the reference price, and thus they do not affect the consumer purchase decision for the current price. The second equation states that, in the absence of reference dynamics, $\frac{d p}{d t}$ is always positive (recall that the second factor on the left-side is positive-see footnote 4). The optimal pricing policy of the inventory is such that the price increases over time; the firm adopts a penetration strategy. Proposing a low price in the first period enables reducing inventory costs through the entire time period by selling more products at the beginning of the selling period. This low initial retail price is possible because it has no impact on the consumer reference price.

\section{Absence of Inventory Cost and Reference Adjustment}

When there is no inventory cost $(h=0)$ and no adjustment of the reference price $(\beta=0)$, then the shadow price of the inventory is null $\left(\lambda_{I}=0\right)$ and the reference price remains constant $(d r / d t=0)$. In this situation, Propositions 1 and 2 write

$$
\begin{aligned}
& -\frac{\partial D}{\partial p} \frac{p}{D}=1, \\
& \frac{d p}{d t}=0 .
\end{aligned}
$$

These results claim that in a dynamic setting when the firm is not constrained by reference and inventory considerations, the pricing policy is that of a standard monopoly. Indeed, the price elasticity of demand is unitary. Also, the price is constant over time because all dynamic effects disappear. In a nutshell, this special case conforms to the pricing of a monopoly in a static situation.

\subsection{Numerical Example}

A numerical example is useful in two ways. First, it provides stronger insights about the relationship between the selling and reference prices. Second, it shows how to compute price in firm applications. 
We work on the classical ${ }^{5}$ linear demand function $D=\alpha-\delta p-\gamma(p-r)$ by posing the parameters $\alpha=1 / 4$ and $\delta=\gamma=1 / 2$, such that $D=1 / 4-p+1 / 2 r$. Reference price dynamics are given by $d r / d t=1 / 2(p-r)$, meaning that $\beta=1 / 2$. Further, the retailer is farsighted, valuing equally present and future profits, that is $\rho=0$. For simplification, the holding cost is low, and may be approximated by 0 , that is $h=0$. In this situation, we have $\pi=p(1 / 4-p+1 / 2 r), \lambda_{I}=0$, and $\lambda_{r}(t)=1 / 2 \int_{t}^{T} e^{-1 / 2(s-t)} p d s$.

With such specification, Proposition 1 writes

$$
p=\frac{1}{8}+\frac{r}{4}+\frac{\lambda_{r}}{4}
$$

linking the optimal relationships between the selling and the reference prices. Proposition 2 reads

$$
\frac{d p}{d t}=\frac{1}{4} \frac{d r}{d t}+\frac{1}{8}\left(\lambda_{r}-p\right)
$$

showing that the selling price must increase if the reference price increases, and that the selling price may increase, even if the reference price decreases.

With a linear demand function, Propositions 1 and 2 provide insights about the optimal relationship and the dynamics of $p$ and $r$. Yet, they show that analyzing inventory is a complex task even in a simple framework, as the model does not provide closed results. Consequently, one may use numerical approximation techniques to characterize the solution of the model with given initial values for $I_{0}$ and $r_{0}$. Yet, the introduction of reference effect with inventory management complexifies the pricing rules. Indeed, it impedes to obtain simple pricing rules, such as penetration, skimming, $\mathrm{U}$, or inverted-U pricing policies.

\section{Conclusion}

This article studied the dynamic pricing of an inventory, of a monopoly operating in a market where consumers are subject to reference-dependence. The modeling in an optimal control framework contributes to existing literature on behavioral dynamic pricing through the following aspects: The pricing rules explicitly consider the impact of current reference prices and inventory levels of the future profits. Finally, it discusses the managerial implications of behavioral pricing strategies of profit-maximizing firms.

Our model builds on the dynamic pricing model with reference price of Chenavaz (2016), by integrating the inventory element. In order to make the model more realistic, it could be generalized with a stochastic demand, as in Cao et al. (2012), or demand or elasticity be time dependent, as in Schlosser (2015), Schlosser (2016), and Güler et al. (2017). Such task, which complexifies the model, is left for future work.

By pointing to the influence of the psychological element, this article enhances the comprehension of inventory pricing. Firms and retailers would benefit from adapting their pricing policies to consumer behavior, thereby becoming more profitable. Further profit-maximizing opportunities

5 See for instance Sorger (1988), Kopalle et al. (1996), Kopalle and Winer (1996), Dye and Yang (2016), Xue et al. (2016), and Hsieh and Dye (2017). 
require future research on the optimal relationships among directly controllable variables (such as the selling price, marketing expenses, or promotion activities) and indirectly controllable variables (such as the reference price, reference quality, or product goodwill).

\section{A Appendix}

\section{A.1 Proof of Equation (9)}

Recall that the dynamic of $\lambda_{I}$ writes in (8)

$$
\frac{d \lambda_{I}}{d t}=\rho \lambda_{I}-\frac{\partial H}{\partial I}=\rho \lambda_{I}+h, \text { with } \lambda_{I}(T)=0 .
$$

Consider the integrating factor $e^{-\rho t}$, such that

$$
\frac{d \lambda_{I}(t) e^{-\rho t}}{d t}=e^{-\rho t}\left(\frac{d \lambda_{I}(t)}{d t}-\rho \lambda_{I}(t)\right)
$$

Because $\frac{d \lambda_{I}(t)}{d t}-\rho \lambda_{I}(t)=h$, then

$$
\begin{aligned}
& \frac{d\left(\lambda(t) e^{-\rho t}\right)}{d t}=h e^{-\rho t}, \\
& \int_{t}^{T} d\left(\lambda(s) e^{-\rho s}\right)=\int_{t}^{T} e^{-\rho s} h d s, \\
& \lambda(T) e^{-\rho T}-\lambda(t) e^{-\rho t}=\int_{t}^{T} e^{-\rho s} h d s .
\end{aligned}
$$

Substituting the transversality condition $\lambda_{I}(T)=0$ yields

$$
\lambda_{I}(t)=-\int_{t}^{T} e^{-\rho(s-t)} h d s,
$$

which completes the proof.

\section{A.2 Proof of Equation (12)}

Recall that the dynamic of $\lambda_{r}$ writes in (11)

$$
\frac{d \lambda_{r}}{d t}=\rho \lambda_{r}-\frac{\partial H}{\partial r}=(\rho+\beta) \lambda_{r}-\left(p+h-\lambda_{I}\right) \frac{\partial D}{\partial r}, \text { with } \lambda_{r}(T)=0 .
$$

Consider the integrating factor $e^{-(\rho+\beta) t}$, such that

$$
\frac{d\left(\lambda_{r}(t) e^{-(\rho+\beta) t}\right)}{d t}=e^{-(\rho+\beta) t}\left(\frac{d \lambda_{r}(t)}{d t}-(\rho+\beta) \lambda_{r}(t)\right)
$$


Because $\frac{d \lambda_{r}(t)}{d t}-(\rho+\beta) \lambda_{r}(t)=-\left(p+h-\lambda_{I}\right) \frac{\partial D}{\partial r}$, then

$$
\begin{aligned}
& \frac{d\left(\lambda_{r}(t) e^{-(\rho+\beta) t}\right)}{d t}=e^{-(\rho+\beta) t}\left[-\left(p+h-\lambda_{I}\right) \frac{\partial D}{\partial r}\right], \\
& \int_{t}^{T} d\left(\lambda_{r}(s) e^{-(\rho+\beta) s}\right)=\int_{t}^{T} e^{-(\rho+\beta) s}\left(-\left(p+h-\lambda_{I}\right) \frac{\partial D}{\partial r}\right) d s, \\
& \lambda_{r}(T) e^{-(\rho+\beta) T}-\lambda_{r}(t) e^{-(\rho+\beta) t}=\int_{t}^{T} e^{-(\rho+\beta) s}\left(-\left(p+h-\lambda_{I}\right) \frac{\partial D}{\partial r}\right) d s .
\end{aligned}
$$

Substituting the transversality condition $\lambda_{r}(T)=0$ yields

$$
\lambda_{r}(t)=\int_{t}^{T} e^{-(\rho+\beta)(s-t)}\left(p+h-\lambda_{I}\right) \frac{\partial D}{\partial r} d s,
$$

which completes the proof.

\section{A.3 Proof of Equation (13b)}

Recall the first-order condition with respect to $p$ (13a)

$$
\begin{gathered}
\frac{\partial H}{\partial p}=0 \Longrightarrow D+p \frac{\partial D}{\partial p}+h D+\beta \lambda_{r}-\frac{\partial D}{\partial p} \lambda_{I}=0, \\
\Longrightarrow(1+h) D+p \frac{\partial D}{\partial p}+\beta \lambda_{r}-\frac{\partial D}{\partial p} \lambda_{I}=0 .
\end{gathered}
$$

The second-order condition with respect to $p$ (13b) states

$$
\begin{aligned}
& \frac{\partial^{2} H}{\partial p^{2}}<0 \Longrightarrow(1+h) \frac{\partial D}{\partial p}+\frac{\partial D}{\partial p}+p \frac{\partial^{2} D}{\partial p^{2}}-\frac{\partial^{2} D}{\partial p^{2}} \lambda_{I}<0, \\
& \Longrightarrow(2+h) \frac{\partial D}{\partial p}+\frac{\partial^{2} D}{\partial p^{2}}\left(p-\lambda_{I}\right)<0, \\
& \Longrightarrow-(2+h) \frac{\partial D}{\partial p}-\left(-\lambda_{I}+p\right) \frac{\partial^{2} D}{\partial p^{2}}>0,
\end{aligned}
$$

which completes the proof.

\section{A.4 Proof of Proposition 2}

Recall the first-order condition (13a)

$$
(1+h) D(p(t), r(t))+p(t) \frac{\partial D(p(t), r(t))}{\partial p}+\beta \lambda_{r}(t)-\frac{\partial D(p(t), r(t))}{\partial p} \lambda_{I}(t)=0 .
$$

Differentiate the first order condition (13a) with respect to $t$ gives

$$
\begin{aligned}
& (1+h)\left(\frac{\partial D}{\partial p} \frac{d p}{d t}+\frac{\partial D}{\partial r} \frac{d r}{d t}\right)+\frac{d p}{d t} \frac{\partial D}{\partial p}+p\left(\frac{\partial^{2} D}{\partial p^{2}} \frac{d p}{d t}+\frac{\partial^{2} D}{\partial p \partial r} \frac{d r}{d t}\right) \\
& +\beta \frac{d \lambda_{r}}{d t}-\left(\left(\frac{\partial^{2} D}{\partial p^{2}} \frac{d p}{d t}+\frac{\partial^{2} D}{\partial p \partial r} \frac{d r}{d t}\right) \lambda_{I}+\frac{\partial D}{\partial p} \frac{d \lambda_{I}}{d t}\right)=0,
\end{aligned}
$$


giving after rearrangement

$$
\begin{aligned}
& \frac{d p}{d t}\left(-(2+h) \frac{\partial D}{\partial p}-\frac{\partial^{2} D}{\partial p^{2}}\left(p-\lambda_{I}\right)\right)=\frac{d r}{d t}\left((1+h) \frac{\partial D}{\partial r}+\frac{\partial^{2} D}{\partial p \partial r}\left(p-\lambda_{I}\right)\right) \\
& +\beta \frac{d \lambda_{r}}{d t}-\frac{\partial D}{\partial p} \frac{d \lambda_{I}}{d t} .
\end{aligned}
$$

Substituting (8) and (11) above provides

$$
\begin{aligned}
\frac{d p}{d t}\left(-(2+h) \frac{\partial D}{\partial p}-\frac{\partial^{2} D}{\partial p^{2}}\left(p-\lambda_{I}\right)\right) & =\frac{d r}{d t}\left((1+h) \frac{\partial D}{\partial r}+\frac{\partial^{2} D}{\partial p \partial r}\left(p-\lambda_{I}\right)\right) \\
& +\beta\left[(\rho+\beta) \lambda_{r}-\left(p+h-\lambda_{I}\right) \frac{\partial D}{\partial r}\right]-\frac{\partial D}{\partial p}\left[\rho \lambda_{I}+h\right],
\end{aligned}
$$

which completes the proof. 


\section{References}

Bakker, M., Riezebos, J. and Teunter, R. H. (2012). Review of inventory systems with deterioration since 2001. European Journal of Operational Research, 221(2): 275-284. https://doi.org/10. 1016/j.ejor.2012.03.004

Cao, P., Li, J. and Yan, H. (2012). Optimal dynamic pricing of inventories with stochastic demand and discounted criterion. European Journal of Operational Research, 217(3): 580-588. https://doi.org/10.1016/j.ejor.2011.09.048

Chenavaz, R. (2016). Dynamic pricing with reference price dependence. Economics: The Open-Access, Open-Assessment E-Journal, 10(2016-22): 1-17. http://dx.doi.org/10.5018/ economics-ejournal.ja.2016-22

Chenavaz, R. (2017). Dynamic quality policies with reference quality effects. Applied Economics, 49(32): 3156-3162. https://doi.org/10.1080/00036846.2016.1254345

Chenavaz, R. Y. and Jasimuddin, S. M. (2017). An analytical model of the relationship between product quality and advertising. European Journal of Operational Research, 263(1): 295-307. https://doi.org/10.1016/j.ejor.2017.05.016

Den Boer, A. V. (2015). Dynamic pricing and learning: Historical origins, current research, and new directions. Surveys in Operations Research and Management Science, 20(1): 1-18. https://doi.org/10.1016/j.sorms.2015.03.001

Dye, C. Y. and Yang, C. T. (2016). Optimal dynamic pricing and preservation technology investment for deteriorating products with reference price effects. Omega, 62: 52-67. https: //doi.org/10.1016/j.omega.2015.08.009

Elmaghraby, W. and Keskinocak, P. (2003). Dynamic pricing in the presence of inventory considerations: Research overview, current practices, and future directions. Management Science, 49(10): 1287-1309. https://doi.org/10.1287/mnsc.49.10.1287.17315

Fibich, G., Gavious, A. and Lowengart, O. (2003). Explicit solutions of optimization models and differential games with nonsmooth (asymmetric) reference-price effects. Operations Research, 51(5): 721-734. https://doi.org/10.1287/opre.51.5.721.16758

Goyal, S. and Giri, B. C. (2001). Recent trends in modeling of deteriorating inventory. European Journal of Operational Research, 134(1): 1-16. https://doi.org/10.1016/S0377-2217(00) 00248-4

Güler, M. G., Akan, M. and Sevim, İ. (2017). Optimal pricing policy with inventory related costs and reference effects. Pamukkale University Journal of Engineering Sciences, 23(4): 451-461. https://www.researchgate.net/publication/319364724_Optimal_pricing_policy_with_ inventory_related_costs_and_reference_effects 
Hsieh, T. P. and Dye, C. Y. (2017). Optimal dynamic pricing for deteriorating items with reference price effects when inventories stimulate demand. European Journal of Operational Research, 262(1): 136-150. https://doi.org/10.1016/j.ejor.2017.03.038

Kalish, S. (1983). Monopolist pricing with dynamic demand and production cost. Marketing Science, 2(2): 135-159. https://doi.org/10.1287/mksc.2.2.135

Kopalle, P. K., Rao, A. G. and Assuncao, J. L. (1996). Asymmetric reference price effects and dynamic pricing policies. Marketing Science, 15(1): 60-85. https://doi.org/10.1287/mksc.15.1. 60

Kopalle, P. K. and Winer, R. S. (1996). A dynamic model of reference price and expected quality. Marketing Letters, 7(1): 41-52. https://doi.org/10.1007/BF00557310

Li, S., Zhang, J. and Tang, W. (2015). Joint dynamic pricing and inventory control policy for a stochastic inventory system with perishable products. International Journal of Production Research, 53(10): 2937-2950. https://doi.org/10.1080/00207543.2014.961206

Lu, L., Zhang, J. and Tang, W. (2016). Optimal dynamic pricing and replenishment policy for perishable items with inventory-level-dependent demand. International Journal of Systems Science, 47(6): 1480-1494. https://doi.org/10.1080/00207721.2014.938784

Mazumdar, T., Raj, S. P. and Sinha, I. (2005). Reference price research: Review and propositions. Journal of Marketing, 69(4): 84-102. https://doi.org/10.1509/jmkg.2005.69.4.84

Nasiry, J. and Popescu, I. (2011). Dynamic pricing with loss-averse consumers and peak-end anchoring. Operations research, 59(6): 1361-1368. https://doi.org/10.1287/opre.1110.0952

Popescu, I. and Wu, Y. (2007). Dynamic pricing strategies with reference effects. Operations Research, 55(3): 413-429. https://doi.org/10.1287/opre.1070.0393

Schlosser, R. (2015). Dynamic pricing with time-dependent elasticities. Journal of Revenue and Pricing Management, 14(5): 365-383. https://doi.org/10.1057/rpm.2015.3

Schlosser, R. (2016). Joint stochastic dynamic pricing and advertising with time-dependent demand. Journal of Economic Dynamics and Control, 73: 439-452. https://doi.org/10.1016/j.jedc.2016. 10.006

Sorger, G. (1988). Reference price formation and optimal pricing strategies. In Feichtinger, G. (Ed.), Optimal Control Theory and Economic Analysis 3, pp. 97-120. Elsevier.

Taudes, A. and Rudloff, C. (2012). Integrating inventory control and a price change in the presence of reference price effects: a two-period model. Mathematical Methods of Operations Research, 75(1): 29-65. https://doi.org/10.1007/s00186-011-0374-1

Xue, M., Tang, W. and Zhang, J. (2016). Optimal dynamic pricing for deteriorating items with reference-price effects. International Journal of Systems Science, 47(9): 2022-2031. https://doi.org/10.1080/00207721.2014.970598 


\section{Economics}

Please note:

You are most sincerely encouraged to participate in the open assessment of this article. You can do so by either recommending the article or by posting your comments.

\section{Please go to:}

http://dx.doi.org/10.5018/economics-ejournal.ja.2018-64

The Editor 